\title{
Editorial
}

Public Health

Published online: November 14, 2015 Genomics

\section{Getting Personal: Accelerating Personalised and Precision Medicine Integration into Clinical Cancer Research and Care in Clinical Trials}

\author{
Denis Horgan ${ }^{a, b}$ Mark Lawler ${ }^{a, b} \quad$ Angela Brand ${ }^{b, c}$ \\ a European Alliance for Personalised Medicine, Brussels, Belgium; ${ }^{b}$ Centre for Cancer Research and Cell Biology, \\ Queen's University Belfast, Belfast, UK; ' Institute for Public Health Genomics, University of Maastricht, Maastricht, \\ The Netherlands
}

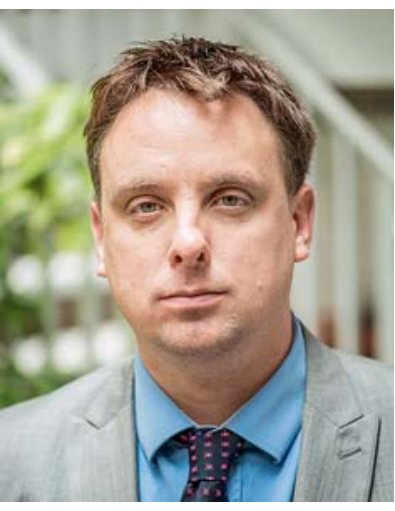

Denis Horgan

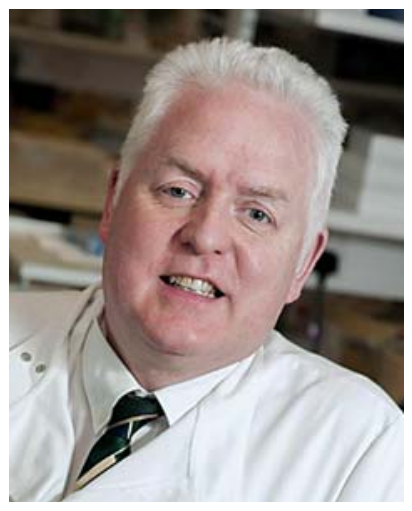

Mark Lawler

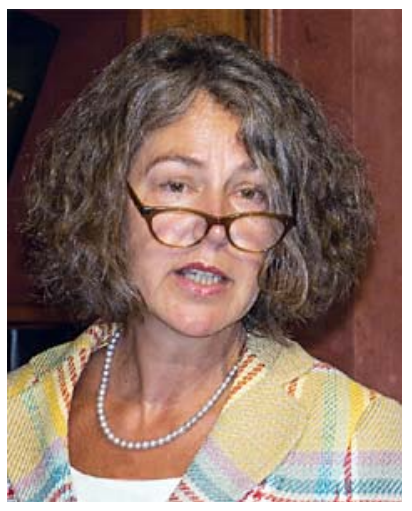

Angela Brand
Personalised and precision medicine (PPM) has the potential to revolutionise not only the treatment but also the prevention of malignancy [1]. Advances such as the successful development of targeted therapies like imatinib mesylate for chronic myeloid leukaemia and Herceptin/trastuzumab for erbB-2-positive breast cancer have changed clinical practice for these disease indications, while a molecular understanding of the human papilloma virus has informed a preventative public health strategy that has underpinned a significant reduction in cervical cancer mortality. However, for every breakthrough therapy like imatinib, there is a succession of 'one-size-fits-all' approaches that deliver minimal or no benefit, despite the billions of euros/dollars and the number of years invested in their development. Increasingly, there is a realisation that we need to facilitate an accelerated 'concept-to-clinic' culture, but in a way that translates preliminary discovery 'hits' into robust but affordable clinical 'wins' for our patients.

Recognising the need to define barriers and to develop putative solutions to the integration of PPM into clinical cancer research and care, the Brussels-based European Alliance for Personalised Medicine (EAPM) assembled a multi-stakeholder panel to address whether a PPM-enabled clinical research/clinical trials strategy can underpin better outcomes for cancer patients. A series of faceto-face meetings and teleconferences informed the identification of a number of key challenges (table 1) that form the basis for this special issue of Public Health Genomics. The authors, who are representative of the diverse stake-
Denis Horgan, Executive Director 
Table 1 Challenges to integrating PPM into cancer health care

- Cancer drug discovery/development model is no longer fit for the purpose

- Traditional clinical trials framework does not support a PPM strategy

- Escalating costs of cancer health care undermine clinical innovation

- Lack of clinically validated biomarkers hampers a PPMenabled approach

- Current European legislative reform threatens clinical cancer research

- Lack of understanding and insufficient patient involvement undermines participation in clinical research

- Absence of cooperation between different stakeholders lessens the effectiveness of PPM implementation

holders in this complex domain, outline these challenges and the potential solutions that will help embed PPM in an evolving cancer care framework.

Lawler and Sullivan highlight the need to empower a paradigm shift in clinical cancer research and its implementation for improved patient care. The clinical trial wheel is broken; we need to develop innovative solutions to fix it. The model of modest increases in benefit may have defined successful clinical trials of the 20th century, but it is no longer pertinent for the PPM-enabled approaches of the molecular era. Clinical trials and clinical cancer research must increasingly be more cooperative (bridging the academia-industry intersect) and deliver benefit - our patients both deserve and demand transformative rather than incremental change. However, this transformative change must be delivered within the real politics of affordable health care systems. Personalised medicine must be progressive, but it must also be pragmatic. The escalating cost of cancer medicines in particular is not sustainable, even for large economies such as the US or Switzerland [2,3]. A cost-versus-value lexicon must be defined, with a specific emphasis on delivering increased benefit from a health, innovation, economic and societal perspective. We need an evidence-based interpretation of therapeutic 'value'. Approaches such as the recently published European Society of Medical Oncology Magnitude of Clinical Benefit Scale (ESMOMCBS) [4] allow us to begin to chart a course for optimal but affordable health care for our cancer patients.

Continuing the theme of delivering transformative benefit, Hollingsworth and Biankin emphasise the need for an innovative clinical trial culture that matches the trial to the patient, rather than finding the patient for the trial. Increasingly, we need to move away from the traditional single-arm approach to a more nuanced multiarm, multi-stage clinical trial algorithm which is supported by a precise molecular stratification strategy. Innovative approaches such as FOCUS4 [5] or NCI-MATCH [6] represent the future: biomarker-informed multi-agent clinical trials. In addition to bridging the academiaindustry intersect, these approaches will increasingly require new partnership models, leveraging international collaboration and promoting multi-pharma collaborative networks such as pre-competitive collaboration.

Crucial to the success of these new clinical trial algorithms is the availability of biomarkers to drive patient stratification and treatment selection. Despite the vast amount of research and the many scientific publications in this area, the number of validated biomarkers that have real clinical application is miniscule. Schneider et al. argue for the need to define precise evidence requirements for clinically applicable biomarkers, setting a defined evidence bar that must be reached for their clinical adoption and reimbursement. Establishing such a framework allows researchers and diagnostic companies to plan their biomarker pipeline appropriately, while providing regulators and payers with the relevant tools to judge clinical applicability.

PPM research and its application is taking place against a background of regulatory uncertainty. The European Commission has undertaken a legislative review of a number of key areas that impact on clinical research. A new Clinical Trials Regulation has replaced the previous Clinical Trial Directive, while proposals for new regulations for data protection and in vitro diagnostics are under review.

The central role of the patient and their engagement in clinical research is also highlighted by Gorini et al., who evaluate the patient's decision-making process, with particular emphasis on participation in phase I clinical trials. Increasingly, the patient must be recognised as an active participant rather than a passive recipient in their care. Facilitating the patient to be involved in both clinical research and its application is crucial to a patient-centred cancer control strategy. Participation in clinical research can be challenging, with issues such as consent, clinical trial randomisation and molecular stratification often proving confusing for the patient. The need to promote clinical research and clinical trial literacy through patient-adapted literature should be encouraged but must include patient input. Linking patient-adapted literature to decision-making tools can also prove helpful. The traditional physician-patient relationship also must change, 
evolving towards an equal, shared decision-making partnership. Implementing these patient-focused changes can promote increased trust and understanding, underpinning enhanced participation in cancer clinical trials.

Lehrach moves us into the (very near) future setting the scene for the virtual clinical trial, describing the deployment of computational models to predict patient responses to drugs in silico, with applications in personalised medicine and drug development and discovery. Such models have the potential to move us beyond the current (albeit biased) therapeutic lottery that doctors and patients are currently forced to play, providing a powerful companion to the real-life clinical trial process, and generating benefits not only for patient welfare but financial incentives too. The route towards a more focused and efficient clinical trial process is described, a process that embraces the irreducible complexity of the 'biological system' that each human being is and which is constantly evolving as more and more information becomes available, improving the accuracy and validity of predictions - a 'future medicine' of today that requires the necessary changes in legislation and regulatory frameworks to support its implementation.

While this special issue has a European focus, cancer is a global disease. Increasingly, we need to examine the challenges that are presented in other parts of the world and the solutions that may help inform the European context. Syme et al. outline the challenges in translating PPM into standard of care in the Canadian context. The Canadian health care system has some structural similarities with that in Europe. Regional networks and province-specific strategies echo the European Union's member state-specific health care policy. However, in cancer, a number of coordinating activities in clinical trials, federated biobanking, and genomics and personalised health empower a nationally attuned strategy. Streamlining of operational issues and the development of multi-stakeholder collaborations across provincial borders have helped underpin a more progressive approach to enhancing cancer research and care.

Integrating PPM into a modern cancer care pathway that unlocks the value of research is challenging. But as Tejpar and Salgado emphasise, we are now establishing the evidence base and the multi-stakeholder partnerships that will drive transformative care for cancer patients. This evidence base will increasingly involve the generation and analysis of real-life data, including patient-reported outcomes. For data to have real benefit, they must have the potential to be shared. Combining data sets can reveal new insights into disease aetiology and response to therapeutic intervention, which can inform the development of better treatments for patients. In this regard, the Global Alliance for Genomics and Health (GA4GH), an international collaboration between researchers, clinicians, patients, biotech and information technology industries and ethicists, is developing infrastructures and technological approaches to ensure the effective and responsible sharing of clinically annotated genomic data for patient benefit [7].

Realising the potential of PPM requires the integration and alignment of multiple stakeholders across the health care system. A dialogue between stakeholders should be initiated early to ensure that innovation and 'real' benefit is rewarded, while proposed therapeutic approaches with a limited potential to deliver better clinical outcomes should 'fail' at an earlier stage, thus limiting costs and reducing patient participation in clinical trials that deliver little or no clinical benefit. Enhancing and maintaining quality-assured clinical trial infrastructures, through organisations such as the European Organisation for Research and Treatment of Cancer (EORTC), must underpin the development of new therapies. Without innovative clinical trials in Europe, patients' access to PPM is at risk, but this innovation must occur within a culture of rigour and transparency, thus ensuring optimal quality care for cancer patients.

Negrouk et al. highlight the challenges that these new regulations pose, arguing that in their current format, a number of these new legislative instruments will have a negative impact on clinical cancer research and care. Regulatory frameworks are not keeping pace with the evolving clinical research landscape and may significantly hamper the successful integration of PPM into health care systems. Requirements for cost-effective new therapeutic interventions should include health technology assessment, which should be harmonised at a European level. Similarly, the use of real-life data and comparative effectiveness research approaches should contribute to the evidence base. Developing a closer and earlier dialogue between researchers, industry, regulators and payers in this rapidly evolving landscape will inform a more progressive approach that balances the patients' rights with the need to improve the health and wealth of European citizens and societies.

This special issue opens a dialogue on the underlying challenges and the solutions required to embed PPM in European cancer health care systems. Over 1.75 million European citizens die from cancer every year while the ageing population will contribute to significant increases in cancer incidence over the next 20 years. Now is the time to deliver focused solutions that will improve outcomes for cancer patients in Europe. 


\section{References}

$>1$ Lawler M, Selby P: Personalized cancer medicine: are we there yet? Oncologist 2013;18: 649-650.

12 Experts in Chronic Myeloid Leukemia: The price of drugs for chronic myeloid leukemia (CML) is a reflection of the unsustainable prices of cancer drugs: from the perspective of a large group of CML experts. Blood 2013; 121:4439-4442.

3 Lawler M, Duffy S, La Vecchia C, Le Chevalier T, Selby PJ, Sullivan R, Johnston PG: America's cancer care crisis - is Europe any better? Lancet 2013;382:1628.
4 Cherny NI, Sullivan R, Dafni U, Kerst JM, Sobrero A, Zielinski C, de Vries EG, Piccart MJ: A standardised, generic, validated approach to stratify the magnitude of clinical benefit that can be anticipated from anti-cancer therapies: the European Society for Medical Oncology Magnitude of Clinical Benefit Scale (ESMO-MCBS). Ann Oncol 2015;26:15471573.
5 Kaplan R, Maughan T, Crook A, Fisher D, Wilson R, Brown L, Parmar M: Evaluating many treatments and biomarkers in oncology: a new design. J Clin Oncol 2013;31:45624568 .

6 McNeil C: NCI-MATCH launch highlights new trial design in precision-medicine era. J Natl Cancer Inst 2015;107:djv193.

7 Lawler M, Siu LL, Rehm HR: All the world's a stage: facilitating discovery science and improved cancer care through the Global Alliance for Genomics and Health. Cancer Discov, in press. 